\title{
Isotope structure of Sm II as an indicator of $r$ - vs. s-process nucleosynthesis
}

\author{
M. Lundqvist ${ }^{1}$, G. M. Wahlgren ${ }^{1}$, and V. Hill ${ }^{2}$ \\ 1 Atomic Astrophysics, Lund Observatory, Lund University, Box 43, 22100 Lund, Sweden \\ e-mail: martin.lundqvist@astro.lu.se \\ 2 GEPI, Observatoire de Paris Meudon, 92125 Meudon Cedex, France
}

Received 11 August 2006 / Accepted 14 November 2006

ABSTRACT

\begin{abstract}
Aims. The isotope composition of samarium provides a previously unexploited means of determining the $r$-versus $s$-process content of e.g. metal-poor stars. The seven stable isotopes of samarium can be divided into two groups, where in one group the $r$-process is the dominant production mechanism, while for the second group it is the $s$-process that is dominant. The center-of-gravity for spectral lines produced by these two groups are slightly shifted with respect to each other, where the heavier and most abundant isotopes are mainly produced by the $r$-process. This relative shift makes the line position dependent upon the content of $r$-and $s$-processed samarium. The isotope composition also affects the width and the shape of the line, making it narrower and slightly more asymmetric when the composition deviates from the solar system composition.

Methods. The behaviour of Sm II lines in stellar spectra is studied using synthetic spectrum techniques with line data accounting for both isotope and hyperfine structure. Isotope shifts in 11 lines of Sm II have been measured from a laboratory spectrum obtained with a Fourier transform spectrometer. Hyperfine structure constants have been taken from the literature.

Results. The new atomic data are applied to the spectrum of the metal-poor halo star CS 31082-001 and suggests that this star contains predominantly $r$-processed material. We also discuss the uncertainties involved in this type of high precision measurement.
\end{abstract}

Key words. atomic data - line: profiles - stars: individual: CS 31082-001 - nuclear reactions, nucleosynthesis, abundances methods: analytical

\section{Introduction}

The study of metal-poor halo stars has produced elemental abundance patterns that have been interpreted as the $r$-process being the highly dominant neutron-capture process active in producing heavy elements in still previous generations of star formation. Extensive elemental abundance analyses of several halo stars have been compared with abundance distributions that represent a scaled solar $r$-process (e.g. Westin et al. 2000; Hill et al. 2002; Sneden et al. 2003). The results to date show that for these few stars, over the atomic number range limited to the lanthanides and some heavier elements, a good agreement is reached, but outside of this range the agreement is poorer. Improvements in atomic data have lead to better agreement in the cases of several elements (Hf: Lundqvist et al. 2005; Os, Ir: Ivarsson et al. 2003). However, model fitting of abundance patterns has represented essentially the only measure of the relative importance of $r$ - and $s$-process nucleosynthesis, since typically no direct measurement is made of the isotope composition. Such data can be obtained by examining the line profiles for transitions in which atomic processes, i.e. isotope shift (IS) and hyperfine structure (hfs), play a key role in shaping line profiles observed at high spectral resolution.

Previous work has attempted to use structure of europium and barium lines as indicators of $r$ - and $s$-process content in metal-deficient halo stars. Europium $(Z=63)$ possesses two stable, odd-numbered isotopes $(\mathrm{A}=151,153)$ and its IS has been investigated for determining an isotope fraction. The relative solar system content of europium produced by the $s$-process is only $5.8 \%$, divided between the two isotopes (Arlandini et al. 1999), which means that it has little impact on the relative contribution of the $r$ - and $s$-process to the isotope fraction. Instead, the aim of the applied method has been to determine whether the $r$-process produced both isotopes in the same relative amount throughout galactic chemical evolutionary history. For each of three metal-poor halo stars Sneden et al. (2002) found an isotope fraction, $\operatorname{fr}\left({ }^{153} \mathrm{Eu}\right)=0.5 \pm 0.1$, which is comparable to the solar system value $\left(\mathrm{fr}\left({ }^{153} \mathrm{Eu}\right)=0.522\right.$, Anders \& Grevesse 1989). This sets constraints on galactic chemical evolution models, forcing them to produce the same isotope mixture as in the Sun early in the history of the galaxy.

Barium has been used to investigate the $r$-versus $s$-process in metal-poor stars. Terrestrial barium is comprised of seven stable isotopes, of which only five have a significant abundance. The composition is heavily dominated by ${ }^{138} \mathrm{Ba}$, which accounts for nearly $72 \%$ of the barium abundance. This isotope is mainly produced by the $s$-process while the odd isotopes $(\mathrm{A}=135,137)$ are to a greater extent produced by the $r$-process. The difference between the $r$-only isotope mixture and a solar mixture of $r$ and $s$ isotopes produces a slight difference in the line shape as the hfs components of the odd-numbered isotopes (accounting for $\sim 18 \%$ of the solar system barium composition) become relatively stronger with a higher fraction of $r$-process produced barium. By measuring the fraction of odd isotopes $\left(f_{\text {odd }}\right)$, i.e. the strength of the line wings, it is, in principle, possible to detect the ratio of $r$-versus $s$-process produced barium. Early results (Magain \& Zhao 1993) argued that no difference in isotope ratios from the solar value exists in the metal-poor star HD 140283 
$\left(f_{\text {odd }}=0.2 \pm 0.1\right)$. Later, Magain (1995) re-analyzed this star giving $f_{\text {odd }}=0.08 \pm 0.06$, which would indicate a pure $s$-process composition for these isotopes. More recent work by Lambert \& Allende Prieto (2002) has challenged the work of Magain and Magain \& Zhao, claiming clear empirical evidence for a solar composition of barium produced by the $r$-process in HD 140283. The value they present is $f_{\text {odd }}=0.30 \pm 0.21$. Both groups base their analysis on observations of a single line, Ba II 24554 , and the extensive error bars do not yet allow for a clear resolution to this problem.

The $s / r$-process ratio has also been investigated by Mashonkina \& Zhao (2005) using the property that hfs affects the odd, $r$-processed isotopes differently in resonance and subordinate lines of Ba II. Their non-LTE calculations of barium abundances for stars of the halo and thick disk may hint at an increase in the $s$-process contribution to barium with time.

Spectral lines of samarium can, through their width, shape and position, provide a means for a more sensitive measurement of the $r$-versus $s$-process content. It is interesting to note that in laboratory spectra taken 70 years ago King (1935) identified many lines of Sm I and Sm II that were double-peaked, some of them with a wavelength splitting as large as $100 \mathrm{~m} \AA$ or more. He explained this line splitting in terms of IS, with the heavier isotopes $(A=152$ and 154$)$ being responsible for the blue peak, but pointed out that high spectral resolution was needed to make further investigations.

Ekeland \& Hauge (1975) used the IS and hfs of two Sm II lines to obtain two ratios of the isotopes in the Sun. Sm II $\lambda 4467$ has no resolvable line structure due to IS but the two odd isotopes $(\mathrm{A}=147,149)$ are affected by hfs. They used this property in a manner similar to the method used to analyze the barium line mentioned earlier (Magain \& Zhao 1993) and derived $f_{\text {odd }}=0.28 \pm 0.07$. The other line they analyzed, Sm II $\lambda 4519$, has IS and since the two heaviest isotopes are well separated from the rest the ratio $\left(\mathrm{Sm}^{152}+\mathrm{Sm}^{154}\right) / \mathrm{Sm}$ was determined to be $0.45 \pm 0.10$. The terrestrial value is 0.495 (Rosman $\&$ Taylor 2004). Ekeland \& Hauge never discussed how different isotopes could be attributed to the $r$ - or $s$-process. Arlandini et al. (1999) created stellar evolutionary models that they claim to reproduce the solar distribution for solely $s$-process nuclei, thereby making it possible to decompose the solar abundance into $r$ - and $s$-process components.

More recently, based on laboratory data for the first three spectra of samarium, Wahlgren (2002) suggested that isotope structure in samarium lines would be useful in investigating the $r$ - vs $s$-process contributions in galactic halo stars. This idea was reiterated by Lawler et al. (2006), who determined new oscillator strengths for a large number of Sm II lines and new abundances in the Sun and three metal-poor stars. They discuss the attribution of samarium to $r$ - and $s$-processing, pointing out the possibility to use this to determine the $r$ - or $s$-process content of metal-poor stars; however, no analysis of Sm II lines was performed for the halo star, owing to the weakness of the lines of interest.

In the current study, the usefulness of samarium as a diagnostic tool to indicate the relative contribution of $r$-versus $s$-process material is presented. New measurements of IS for $11 \mathrm{Sm} \mathrm{II} \mathrm{lines}$ in a laboratory spectrum obtained with a Fourier transform spectrometer are presented, including a discussion of the uncertainties involved in the results and the effect of hfs on the two odd isotopes. The results of the laboratory measurements are applied to the metal-poor halo star CS 31082-001, which from earlier work is thought to have a large $r$-process enhanced abundance of the heavier elements (Hill et al. 2002). The results of the isotopic composition of samarium in CS 31082-001 tend to agree with previous indications of $r$-process enhancement. Higher quality spectra than are currently available are, however, required to place tighter constraints on our results.

\section{Atomic data}

The laboratory data used here were obtained using the Lund UV Fourier transform spectrometer (FTS) and cover the wavenumber range $12800-55000 \mathrm{~cm}^{-1}$ over three instrument setups. This data set was utilized by Wahlgren (2002) and was described by Biémont et al. (2003) in determining branching fractions and oscillator strengths in Sm III. The light source was a hollowcathode lamp with an iron cathode and a piece of samarium inserted into the $10 \mathrm{~mm}$ diameter hole. The carrier gas was neon at a pressure of 1.6 Torr and the lamp was run at a current of $600 \mathrm{~mA}$. For the present study only one of the spectral regions was utilized, that between $12800-24000 \mathrm{~cm}^{-1}$, since the most interesting Sm II lines available in both laboratory and stellar spectra are found in this region. The stellar spectrum also becomes more crowded at higher wavenumbers, making it more difficult to identify unblended lines.

The linear wavenumber scale of the FTS spectrum comes from a single mode He-Ne control laser. The scale is accurate to a few parts in $10^{5}$ and the uncertainties arise from the effects of the finite aperture size and imperfect alignment of the light source and the control laser (Learner \& Thorne 1988). The wavenumber scale was placed onto an absolute scale through a correction factor $(k)$, with the corrected wavenumber $\left(\sigma_{\text {corr }}\right)$ being derived from

$\sigma_{\text {corr }}=(1+k) \sigma_{\mathrm{obs}}$

where $\sigma_{\mathrm{obs}}$ is the observed wavenumber. The calibration is performed using Fe I lines recommended by Learner \& Thorne (1988), having a very high wavenumber accuracy. Ten lines with small wavenumber uncertainties $\left(\Delta \sigma<1.0 \times 10^{-4} \mathrm{~cm}^{-1}\right)$, spread over the full expanse of the spectrum were chosen for the calibration. These lines were fitted with a Voigt profile and an average correction factor was calculated. The $1 \sigma$ uncertainty in $k$ was determined to be $0.0013 \mathrm{~cm}^{-1}$.

Unfortunately, the same Fe I lines could not be used for wavelength calibration in the stellar spectrum because of the difference in excitation conditions. However, five Fe I lines were found near the Sm II lines in both the laboratory and the stellar spectrum (see Table 1). These lines were all too weak for wavenumber calibration of the laboratory spectrum, but when comparing their wavenumber to Learner \& Thorne (1988) after the calibration they were all found to be within $0.002 \mathrm{~cm}^{-1}$, translating into an uncertainty of less than $1 \mathrm{~m} \AA$ in the observed wavelength region. Air wavelengths were calculated using the formalism of Edlén (1966).

\subsection{Isotope shift and hyperfine structure in Sm II lines}

In this work we refer to the isotope shift (IS) as the difference in the energy of an atomic level between two isotopes, and the isotope shift of a transition, or transition isotope shift (TIS), to be the shift of the difference in the upper and lower levels between two isotopes. Thus the IS refers to an energy level while the TIS is associated with the spectral line. The large IS of some samarium levels leads to visible line structure in our laboratory spectrum. Two of the lines having the largest shifts are presented in Figs. 1 and 2. The Sm II lines with this large a TIS have two 
Table 1. Fe I lines used for setting the wavelength scale in the stellar spectrum and determining the rotational velocity.

\begin{tabular}{lcc}
\hline \hline$\lambda_{\text {air }}(\AA)$ & Ref $^{a}$ & $\log g f^{b}$ \\
\hline 4592.6512 & LT & -2.449 \\
4602.9409 & LT & -2.250 \\
4691.412 & TW & -1.450 \\
4710.2834 & LT & -1.612 \\
5049.8196 & LT & -1.420 \\
5051.635 & TW & -2.795 \\
5068.7657 & LT & -1.055 \\
5098.6959 & LT & -2.026 \\
5107.448 & TW & -3.087 \\
5107.641 & TW $^{c}$ & -2.418 \\
5110.413 & TW $^{-3.760}$ \\
\hline
\end{tabular}

${ }^{a}$ TW: This work; LT: Learner \& Thorne (1988); ${ }^{b}$ Kurucz \& Bell (1995); ${ }^{c}$ blended with Nd II line in stellar spectrum.

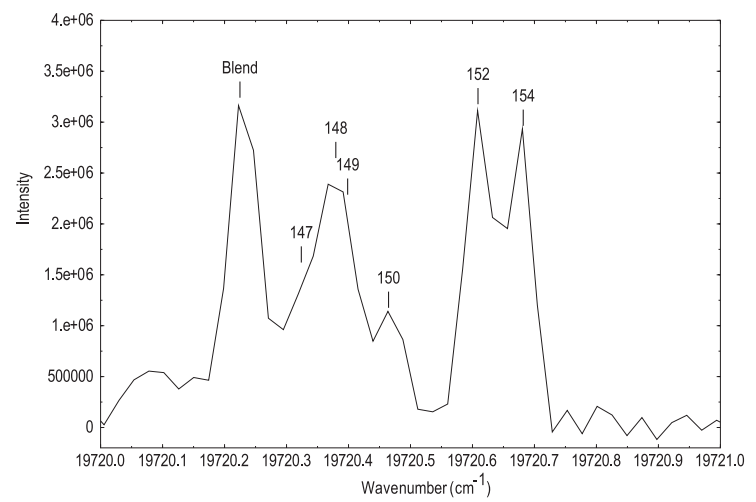

Fig. 1. FTS-spectrum of the line Sm II $\lambda 5069$. Isotope positions are labeled with those for odd-numbered isotopes representing the center-ofgravity location. An unidentified blending feature is also labeled.

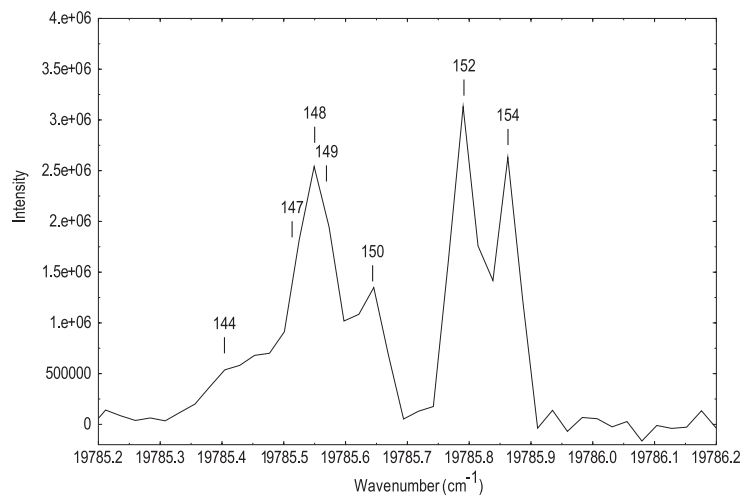

Fig. 2. FTS spectrum of the line Sm II 15053 . Isotope positions are labeled.

main features: the double peak at higher wavenumber is predominantly attributed to isotopes ${ }^{152} \mathrm{Sm}$ and ${ }^{154} \mathrm{Sm}$, but can also be slightly affected by the presence of odd-isotope lines when the hfs is large, while the other, broader feature contains the isotopes ${ }^{144} \mathrm{Sm}$ and ${ }^{147-150} \mathrm{Sm}$. The two odd-numbered isotopes are both affected by hfs. The $\mathrm{A}=144$ isotope is too weak to form a peak but is seen as a hump in Fig. 2.

The wavenumbers of the five even-numbered isotopes were measured by simultaneously fitting Voigt profiles to all peaks. For lines for which no previous data exist, the center-of-gravity wavenumber of the odd isotopes has been estimated by comparison with the lines where the odd isotope positions are measured
Table 2. Comparison of transition isotope shift in Sm II, measured relative to ${ }^{154} \mathrm{Sm}$.

\begin{tabular}{|c|c|c|}
\hline \multirow{2}{*}{$\begin{array}{c}\text { Isotope } \\
\text { A }\end{array}$} & \multicolumn{2}{|c|}{ TIS $\left({ }^{154} \mathrm{Sm}-{ }^{\mathrm{A}} \mathrm{Sm}\right)\left(\mathrm{cm}^{-1}\right)$} \\
\hline & This work & Villemoes et al. \\
\hline$\lambda_{\text {air }}=$ & $5052.751 \AA$ & $5786.98 \AA$ \\
\hline \multicolumn{3}{|c|}{$E_{\mathrm{upper}}=30879.740 \mathrm{~cm}^{-1}$} \\
\hline 148 & 0.311 & 0.312 \\
\hline 150 & 0.219 & 0.214 \\
\hline 152 & 0.072 & 0.071 \\
\hline$\lambda_{\text {air }}=$ & $5069.447 \AA^{a}$ & $5831.02 \AA$ \\
\hline \multicolumn{3}{|c|}{$E_{\text {upper }}=29934.800 \mathrm{~cm}^{-1}$} \\
\hline 148 & 0.303 & 0.294 \\
\hline 150 & 0.204 & 0.202 \\
\hline 152 & 0.068 & 0.067 \\
\hline$\lambda_{\text {air }}=$ & $5103.086 \AA$ & $5897.39 \AA \& \& 563.22 \AA$ \\
\hline \multicolumn{3}{|c|}{$E_{\text {upper }}=28997.140 \mathrm{~cm}^{-1}$} \\
\hline 148 & 0.303 & $0.281 \& 0.282$ \\
\hline 150 & 0.198 & $0.193 \& 0.194$ \\
\hline 152 & 0.065 & $0.064 \& 0.064$ \\
\hline$\lambda_{\text {air }}=$ & $5104.472 \AA$ & $5965.7 \AA$ \\
\hline \multicolumn{3}{|c|}{$E_{\text {upper }}=27631.180 \mathrm{~cm}^{-1}$} \\
\hline 148 & 0.276 & 0.267 \\
\hline 150 & 0.207 & 0.183 \\
\hline 152 & 0.060 & 0.061 \\
\hline
\end{tabular}

${ }^{a}$ Blended on low wavenumber side of profile.

earlier. Where the hfs is narrow a test was conducted, inserting Voigt profiles in the fit for the lines corresponding to the two odd-numbered isotopes. In these cases the results for the odd isotope positions agreed to within $0.01 \mathrm{~cm}^{-1}$ with the estimation. In Table 4 we present wavenumbers and wavelengths for each isotope of these 11 lines. The TIS for each isotope is measured as the wavenumber difference relative to ${ }^{154} \mathrm{Sm}$. The typical uncertainty of the wavenumber difference between the even isotopes is estimated to be about $0.003 \mathrm{~cm}^{-1}$.

There is extensive earlier laboratory work done on IS and hfs in Sm II. The results of Young et al. (1987), Rao et al. (1990), Villemoes et al. (1995), Beiersdorf et al. (1995) and Masterman et al. (2003) have been utilized here. Using collinear laser ion-beam spectroscopy, Beiersdorf et al. (1995) investigated the $J$-dependence of the IS in the fine-structure multiplet $4 \mathrm{f}^{6}\left({ }^{7} \mathrm{~F}\right) 5 \mathrm{~d}^{8} \mathrm{H}_{J}$ using 18 optical transitions. The IS of the $4 \mathrm{f}^{6}\left({ }^{7} \mathrm{~F}\right) 5 \mathrm{~d}$ configuration is very small, $\mathrm{IS}_{\mathrm{A}=154-148} \leq 0.005 \mathrm{~cm}^{-1}$ (Villemoes et al. 1995), or negligible (Rao et al. 1990) and can, especially in the case of stellar applications, be ignored. Villemoes et al. (1995) measured the IS from lines originating from the same upper levels as our lines $\lambda \lambda 5053,5069,5103$ and 5104. The lower levels of these lines are $4 \mathrm{f}^{6}\left({ }^{7} \mathrm{~F}\right) 5 \mathrm{~d}$ states, but not ${ }^{8} \mathrm{H}$. The TIS of these four lines coincides closely with the TIS of the lines measured by us for all isotopes, as can be seen in Table 2. From this close coincidence we conclude that the lower level of the lines measured by us also has a similarly small IS. Using the IS of the lines in Beiersdorf et al. (1995) and the assumption of an IS of zero for $4 \mathrm{f}^{6}\left({ }^{7} \mathrm{~F}\right) 5 \mathrm{~d}^{8} \mathrm{H}_{J}$, it is possible to derive the IS of the upper levels and further, using the measurements of Masterman et al. (2003), calculate the IS for all seven isotopes for a large number of levels. We use this approach to get the level IS for a number of our chosen transitions. In two cases the lines we chose to measure, shown in Table 4, can be directly compared with Masterman et al. (2003) and in four cases they can be compared to the TIS derived from the level IS. The close agreement tells us that our measurement technique, 


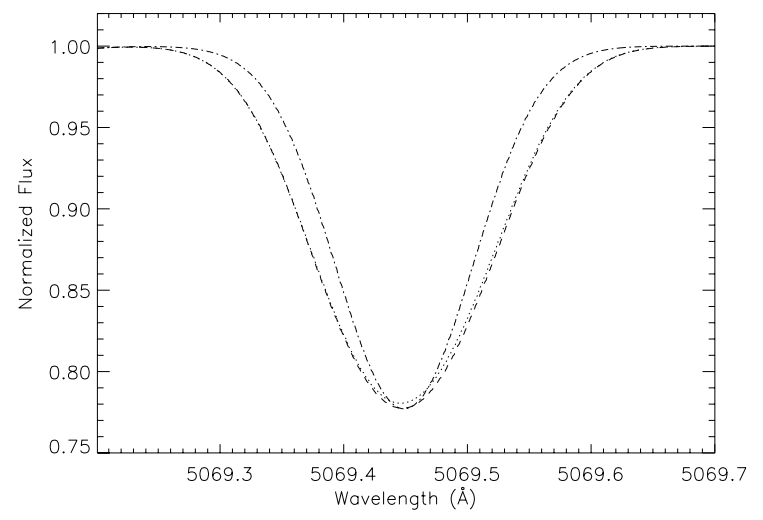

Fig. 3. Synthetic spectra of Sm II $\lambda 5069$ showing the impact of structure. The dotted profile includes hfs and IS, the dashed include only IS and the dot-dashed shows the profile without structure. The difference in abundance between the lines including structure and the line without is 0.1 dex.

though not as accurate as laser techniques, is sufficiently accurate for lines observed at the resolution of stellar spectra. In the other five cases the IS of the upper level is the same as the TIS, since the lower level is $4 \mathrm{f}^{6}\left({ }^{7} \mathrm{~F}\right) 5 \mathrm{~d}^{8} \mathrm{H}_{J}$, which is assumed to have zero IS.

Masterman et al. (2003) measured the magnetic dipole, $A$, and the electric quadrupole, $B$, hyperfine constants for all corresponding levels in their line sample, including all levels for our lines found shortward of $\lambda 4716 . A$ and $B$ constants for the upper level of all lines in our line sample longward of $\lambda 4716$ and the lower level of the $\lambda 5069$ line can be found in the literature (Villemoes et al. 1995; Young et al. 1987). The only level in the $4 \mathrm{f}^{6}\left({ }^{7} \mathrm{~F}\right) 5 \mathrm{~d}^{8} \mathrm{H}_{J}$ configuration with measured $A$ and $B$ constants is for $J=15 / 2$. Figure 3 shows the difference in a synthetic stellar spectrum for this line with and without hfs included. The synthetic spectra are calculated using the model described in Sect. 4, but, for sake of clarity, with a further 1 dex enhancement in the samarium abundance. The difference in this particular line is small, but since the hfs is varying between different levels it cannot be ignored and therefore the lines at $\lambda \lambda 4720$, 5053, 5103 and 5104 have not been used since no hfs constants for their lower levels are available. Included in the figure is also a synthetic spectrum of the line treated without structure, which yields a 0.1 dex lower abundance when matching line depths and is considerably narrower than the line when including its structure. The hfs of the odd Sm II isotopes is included in the line lists using the program LINESTRUC (Wahlgren 2005).

\section{Determining the $r$ - vs. s-process content in a star}

In Table 3 the solar relative abundance and attribution to $r$ - or $s$-process of each isotope is listed. As can be seen, the ${ }^{152} \mathrm{Sm}$ and ${ }^{154} \mathrm{Sm}$ isotopes are dominated by the $r$-process, while ${ }^{148} \mathrm{Sm}$ and ${ }^{150} \mathrm{Sm}$ are only produced by the s-process. ${ }^{147} \mathrm{Sm}$ and ${ }^{149} \mathrm{Sm}$ are both produced to a large extent by the $r$-process and together they contribute approximately a third of the $r$-processed samarium. ${ }^{144} \mathrm{Sm}$ is comparably weak and thereby bears a relatively small impact on the resulting spectral lines. The numbers describing the portion of an isotope produced by $r$ - or $s$-process are taken from the stellar model of Arlandini et al. (1999). Their stellar model results are chosen instead of their classical model results because of their assessment of overproduction of certain $s$-only isotopes by the classical model. The difference between
Table 3. Samarium isotopic composition.

\begin{tabular}{lcc}
\hline \hline A & ${\text { Solar }(\%)^{a}}^{a}(r / s)$ Ratio $^{b}$ \\
\hline 144 & 3.07 & $p^{c}$ \\
147 & 14.99 & $79.3 / 20.7$ \\
148 & 11.24 & $0 / 100$ \\
149 & 13.82 & $87.5 / 12.5$ \\
150 & 7.38 & $0 / 100$ \\
152 & 26.74 & $77.1 / 22.9$ \\
154 & 22.75 & $99.2 / 0.8$ \\
Total & 100 & $67.2 / 29.8$ \\
\hline
\end{tabular}

${ }^{a}$ Rosman \& Taylor (2004); ${ }^{b}$ Arlandini et al. (1999); ${ }^{c}$ Produced by p-process (Anders \& Grevesse 1989).

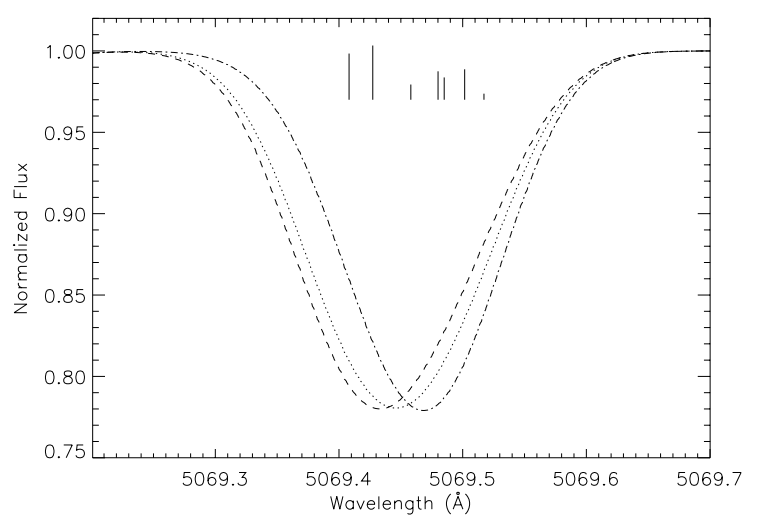

Fig. 4. Synthetic spectra of line Sm II $\lambda 5069$ for the cases of solar isotope mixture (dotted), $r$-only mixture (dashed) and $s$-only mixture (dotdashed). To highlight the line shift, the samarium abundance has been altered for the different spectra to match their line depth. The marks indicate the center position for each of the seven isotopes, with ${ }^{154} \mathrm{Sm}$ being the component at the shortest wavelength.

the classical and stellar models in the case of samarium is minute and, in our case, not detectable.

A line becomes shifted relative to its position for the solar system isotope mixture if its mixture is further dominated by either the $r$ - or $s$-process. In Fig. 4 the comparison between a solar system isotope mixture and mixtures containing only that portion produced by the $r$-process or the $s$-process is shown. The main body of the line becomes shifted, its width (FWHM) is decreased and its shape is slightly altered as the isotope mixture deviates from that of a line reflecting the solar system isotope mixture. The shift of the line from the solar system isotope mixture to the pure $r$-process case is $\sim-13 \mathrm{~m} \AA$ for Sm II $\lambda 5069$. When changing from the solar system mixture to the pure $s$-process case the shift is $\sim+22 \mathrm{~m} \AA$ and the line becomes almost $30 \mathrm{~m} \AA$ narrower. These effects, though small, can in principle be used to investigate the relative abundance of samarium produced by the $r$ - and $s$-processes in i.e. metal-poor halo stars.

All four lines longward of $5000 \AA$ with shifts TIS $_{\mathrm{A}=154-148} \sim$ $0.300 \mathrm{~cm}^{-1}$ which are presented here are visible in our lower $\mathrm{S} / \mathrm{N}$ spectrum of CS 31082-001 (see Sect. 4), although weakly ( $\sim 3 \%$ depth from continuum). Stronger lines are found at bluer wavelengths; however, their TIS is smaller, typically TIS $_{\mathrm{A}=154-148} \leq 0.200 \mathrm{~cm}^{-1}$, making them more difficult to use. These are found in our high-S/N stellar spectrum.

To quantify the small line shifts required for this analysis the wavelength scale of the stellar spectrum must be very accurately determined. Due to their ubiquitous presence, iron lines having high accuracy laboratory wavelengths are found in close 
Table 4. Wavelengths and isotope shifts for select transitions of Sm II.

\begin{tabular}{|c|c|c|c|c|c|c|c|}
\hline \multirow{2}{*}{$\begin{array}{r}\lambda_{\text {air }}(\AA)^{a} \\
\log g f^{b}\end{array}$} & \multirow{2}{*}{$\begin{array}{r}E_{\text {up }}\left(\mathrm{cm}^{-1}\right) \\
E_{\text {low }}\left(\mathrm{cm}^{-1}\right)\end{array}$} & \multirow{2}{*}{$\begin{array}{l}J_{\text {up }} \\
J_{\text {low }}\end{array}$} & \multirow[t]{2}{*}{$\mathrm{A}$} & \multirow{2}{*}{$\begin{array}{c}\sigma \\
\left(\mathrm{cm}^{-1}\right)\end{array}$} & \multirow{2}{*}{$\begin{array}{l}\lambda_{\text {air }} \\
(\AA)\end{array}$} & \multicolumn{2}{|c|}{ Shift $\left(\mathrm{cm}^{-1}\right)$} \\
\hline & & & & & & This work & Earlier work \\
\hline 4591.82 & 23260.950 & 3.5 & 144 & 21771.632 & 4591.846 & 0.190 & $0.193^{c}$ \\
\hline \multirow[t]{6}{*}{-1.120} & 1489.160 & 3.5 & 147 & & & & 0.152 \\
\hline & & & 148 & 21771.695 & 4591.833 & 0.127 & 0.132 \\
\hline & & & 149 & & & & 0.122 \\
\hline & & & 150 & 21771.723 & 4591.827 & 0.099 & 0.092 \\
\hline & & & 152 & 21771.799 & 4591.811 & 0.023 & 0.027 \\
\hline & & & 154 & 21771.822 & 4591.806 & 0 & 0 \\
\hline \multirow{7}{*}{$\begin{array}{r}4593.55 \\
-0.83\end{array}$} & 24816.280 & 5.5 & 144 & 21763.414 & 4593.580 & 0.267 & $0.275^{d}$ \\
\hline & 3052.650 & 5.5 & 147 & & & & 0.212 \\
\hline & & & 148 & 21763.498 & 4593.563 & 0.183 & 0.184 \\
\hline & & & 149 & & & & 0.170 \\
\hline & & & 150 & 21763.560 & 4593.549 & 0.121 & 0.128 \\
\hline & & & 152 & 21763.644 & 4593.532 & 0.037 & 0.040 \\
\hline & & & 154 & 21763.681 & 4593.524 & 0 & 0 \\
\hline \multirow{7}{*}{$\begin{array}{r}4595.29 \\
-0.50\end{array}$} & 25664.970 & 6.5 & 144 & 21755.197 & 4595.315 & 0.168 & $0.162^{c}$ \\
\hline & 3909.620 & 6.5 & 147 & & & & 0.127 \\
\hline & & & 148 & 21755.262 & 4595.302 & 0.103 & 0.110 \\
\hline & & & 149 & & & & 0.104 \\
\hline & & & 150 & 21755.293 & 4595.295 & 0.073 & 0.077 \\
\hline & & & 152 & 21755.352 & 4595.283 & 0.014 & 0.22 \\
\hline & & & 154 & 21755.366 & 4595.280 & 0 & 0 \\
\hline \multirow{7}{*}{$\begin{array}{r}4604.17 \\
-1.390\end{array}$} & 22039.980 & 2.5 & 144 & 21713.189 & 4604.206 & 0.271 & $0.302^{d}$ \\
\hline & 326.640 & 1.5 & 147 & & & & 0.234 \\
\hline & & & 148 & 21713.261 & 4604.191 & 0.200 & 0.203 \\
\hline & & & 149 & & & & 0.187 \\
\hline & & & 150 & 21713.325 & 4604.177 & 0.135 & 0.140 \\
\hline & & & 152 & 21713.418 & 4604.157 & 0.042 & 0.044 \\
\hline & & & 154 & 21713.460 & 4604.148 & 0 & 0 \\
\hline \multirow{7}{*}{$\begin{array}{r}4693.63 \\
-1.48\end{array}$} & 22788.680 & 3.5 & 144 & 21299.335 & 4693.669 & 0.286 & $0.307^{c}$ \\
\hline & 1489.160 & 3.5 & 147 & & & & 0.238 \\
\hline & & & 148 & 21299.427 & 4693.649 & 0.194 & 0.206 \\
\hline & & & 149 & & & & 0.191 \\
\hline & & & 150 & 21299.497 & 4693.633 & 0.123 & 0.143 \\
\hline & & & 152 & 21299.580 & 4693.615 & 0.041 & 0.044 \\
\hline & & & 154 & 21299.621 & 4693.606 & 0 & 0 \\
\hline \multirow{7}{*}{$\begin{array}{r}4715.27 \\
-1.46\end{array}$} & 22039.980 & 2.5 & 144 & 21201.582 & 4715.310 & 0.290 & $0.301^{c}$ \\
\hline & 838.220 & 2.5 & 147 & & & & 0.233 \\
\hline & & & 148 & 21201.674 & 4715.289 & 0.198 & 0.202 \\
\hline & & & 149 & & & & 0.187 \\
\hline & & & 150 & 21201.735 & 4715.276 & 0.137 & 0.140 \\
\hline & & & 152 & 21201.830 & 4715.255 & 0.042 & 0.044 \\
\hline & & & 154 & 21201.872 & 4715.245 & 0 & 0 \\
\hline \multirow{7}{*}{$\begin{array}{r}4719.85 \\
-1.24\end{array}$} & 21507.870 & 1.5 & 144 & 21181.041 & 4719.883 & 0.250 & \\
\hline & 326.640 & 1.5 & 147 & 21181.080 & 4719.874 & 0.21 & \\
\hline & & & 148 & 21181.124 & 4719.864 & 0.165 & \\
\hline & & & 149 & 21181.150 & 4719.859 & 0.14 & \\
\hline & & & 150 & 21181.187 & 4719.850 & 0.103 & \\
\hline & & & 152 & 21181.258 & 4719.835 & 0.032 & \\
\hline & & & 154 & 21181.290 & 4719.827 & 0 & \\
\hline 5052.75 & 30879.740 & 6.5 & 144 & 19785.429 & 5052.816 & 0.433 & \\
\hline \multirow[t]{6}{*}{-0.16} & 11094.060 & 7.5 & 147 & 19785.495 & 5052.799 & 0.368 & \\
\hline & & & 148 & 19785.551 & 5052.784 & 0.311 & \\
\hline & & & 149 & 19785.575 & 5052.778 & 0.288 & \\
\hline & & & 150 & 19785.643 & 5052.761 & 0.219 & \\
\hline & & & 152 & 19785.791 & 5052.723 & 0.072 & \\
\hline & & & 154 & 19785.862 & 5052.705 & 0 & \\
\hline
\end{tabular}


Table 4. continued.

\begin{tabular}{|c|c|c|c|c|c|c|c|}
\hline \multirow{2}{*}{$\begin{array}{r}\lambda_{\text {air }}(\AA)^{a} \\
\log g f^{b}\end{array}$} & \multirow{2}{*}{$\begin{array}{r}E_{\text {up }}\left(\mathrm{cm}^{-1}\right) \\
E_{\text {low }}\left(\mathrm{cm}^{-1}\right)\end{array}$} & \multirow{2}{*}{$\begin{array}{l}J_{\text {up }} \\
J_{\text {low }}\end{array}$} & \multirow[t]{2}{*}{$\bar{A}$} & \multirow{2}{*}{$\begin{array}{c}\sigma \\
\left(\mathrm{cm}^{-1}\right)\end{array}$} & \multirow{2}{*}{$\begin{array}{l}\lambda_{\text {air }} \\
(\AA)\end{array}$} & \multicolumn{2}{|c|}{ Shift $\left(\mathrm{cm}^{-1}\right)$} \\
\hline & & & & & & This work & Earlier work \\
\hline $5069.47^{e}$ & 29934.800 & 5.5 & 147 & 19720.315 & 5069.500 & 0.363 & \\
\hline \multirow[t]{5}{*}{-0.31} & 10214.380 & 6.5 & 148 & 19720.375 & 5069.484 & 0.303 & \\
\hline & & & 149 & 19720.395 & 5069.479 & 0.283 & \\
\hline & & & 150 & 19720.474 & 5069.459 & 0.204 & \\
\hline & & & 152 & 19720.610 & 5069.424 & 0.068 & \\
\hline & & & 154 & 19720.678 & 5069.406 & 0 & \\
\hline 5103.09 & 28997.140 & 4.5 & 144 & 19590.299 & 5103.145 & 0.397 & \\
\hline \multirow[t]{6}{*}{-0.35} & 9406.630 & 5.5 & 147 & 19590.355 & 5103.130 & 0.340 & \\
\hline & & & 148 & 19590.393 & 5103.121 & 0.303 & \\
\hline & & & 149 & 19590.425 & 5103.112 & 0.270 & \\
\hline & & & 150 & 19590.497 & 5103.093 & 0.198 & \\
\hline & & & 152 & 19590.630 & 5103.059 & 0.065 & \\
\hline & & & 154 & 19590.696 & 5103.042 & 0 & \\
\hline 5104.47 & 27631.180 & 2.5 & 144 & 19584.965 & 5104.535 & 0.399 & \\
\hline \multirow[t]{6}{*}{-0.65} & 8046.000 & 3.5 & 147 & 19585.045 & 5104.514 & 0.319 & \\
\hline & & & 148 & 19585.088 & 5104.503 & 0.276 & \\
\hline & & & 149 & 19585.115 & 5104.496 & 0.249 & \\
\hline & & & 150 & 19585.157 & 5104.485 & 0.207 & \\
\hline & & & 152 & 19585.304 & 5104.447 & 0.060 & \\
\hline & & & 154 & 19585.364 & 5104.431 & 0 & \\
\hline
\end{tabular}

${ }^{a}$ Center of gravity; ${ }^{b}$ Lawler et al. (2006); ${ }^{c}$ Beiersdorf et al. (1995) and Masterman et al. (2003); ${ }^{d}$ Masterman et al. (2003); ${ }^{e}$ blended at long wavelength end in FTS spectrum.

proximity to the Sm II lines, and serve to set an accurate wavelength scale in the stellar spectrum though the Fe I lines which are strong in the star are weak in the laboratory spectrum. Where iron lines were not found in the laboratory spectrum or were suspected to be blended, additional lines from Learner \& Thorne (1988) were incorporated. Table 1 presents lines of Fe I used for wavelength comparison with the Sm II lines.

\section{The case of CS 31082-001}

CS 31082-001 is a metal-poor halo giant with a metallicity of $[\mathrm{Fe} / \mathrm{H}]=-2.9$. The model atmosphere used by us is an ATLAS12 model with the same atmospheric parameters as Hill et al. (2002), $T_{\text {eff }}=4825 \mathrm{~K}, \log g=1.5$ and microturbulent velocity $V_{\xi}=1.8 \mathrm{~km} \mathrm{~s}^{-1}$. The macroturbulent velocity $V_{\text {macro }}=$ $2.8 \mathrm{~km} \mathrm{~s}^{-1}$ was determined using $\mathrm{Fe} \mathrm{I}$ lines. The iron abundance, with a $1 \sigma$ uncertainty, was derived from the Fe I lines in Table 1 to be $\log \epsilon(\mathrm{Fe})=4.79 \pm 0.11$ on a scale where $\log \epsilon(H)=12.00$. This is a higher value than the result of Hill et al. (2002) $(\log \epsilon(\mathrm{Fe})=4.60 \pm 0.13)$, although within their mutual error bars.

We have used two sets of data, both obtained using the UVES echelle spectrograph at the ESO VLT facility. The first set has a resolving power of $\sim 75000$ and a $\mathrm{S} / \mathrm{N}$ per pixel of up to 250 . However, for a number of Sm II lines the $\mathrm{S} / \mathrm{N}$ is only about half this value. The wavelength calibration is performed with ThAr lamp frames and is corrected for radial velocity shifts. This data set and the star are more thoroughly described in Hill et al. (2002). The second dataset has a resolving power of 80000 and a S/N per pixel of up to 600 (Plez et al. 2004). The total exposure time is $17 \mathrm{~h}$ and it is also wavelength calibrated using the Th-Ar lamp.

The spectrum analysis was conducted by fitting the observed spectrum with synthetic spectrum calculations made using SYNTHE (Kurucz 1993). Line list data for all lines except
Fe I and Sm II were obtained from the compilation of Kurucz \& Bell (1995). The $g f$ values for the Sm II lines are taken from Lawler et al. (2006) and the wavelength data of the Fe I lines are as described in Table 1. For the Sm II lines where laser measurements from Masterman et al. (2003) existed or the line shift could be extracted according to the method described in Sect. 3, that shift was used. The absolute wavelength was set by using our FTS measurement of the wavelength for the ${ }^{154} \mathrm{Sm}$ isotope.

For the determination of the wavelength scale synthetic spectra were calculated to fit each iron line in the vicinity of the Sm II lines, using a computational resolution (RES) of $9 \times 10^{6}$. The spacing between the calculated points in the synthetic spectrum is given by $\lambda /$ RES. This adds $\lambda / \operatorname{RES} \times 1 / 2$ to the uncertainty of the wavelength. When fitting the iron lines the line troughs were avoided because some lines appear slightly skewed in this portion of the line profile, and instead, fitting the halfwidth points provided good fits. When possible, several Fe I lines were used to determine the wavelength scale. The wavelength calibration was found to be within the accuracy of the Fe I line list $(1 \mathrm{~m} \AA)$. The continuum was placed by eye using a spectral range of about $10 \AA$ around the line of interest. The continuum set by us never departed by more than $2 \%$ from the result of the reduction pipeline and the departure was in all cases constant over the $10 \AA$ range. The uncertainty of the continuum placement does not affect the resulting abundance by more than \pm 0.02 dex and it does not noticably affect the analysis of the line shift.

Synthetic spectra were calculated for pure $s$, pure $r$ and solar system isotopic ratios and compared with the stellar spectrum. In Table 3 the solar isotope composition and the fraction of each isotope produced by the $s$ - or $r$-process are presented. ${ }^{144} \mathrm{Sm}$ is a pure $p$-process isotope and due to our lack of knowledge of when this isotope is produced we keep its abundance constant when changing the $r$ - and $s$-process isotopes. The $p$-process contribution to the other isotopes is thought to be small (Arlandini et al. 1999). 


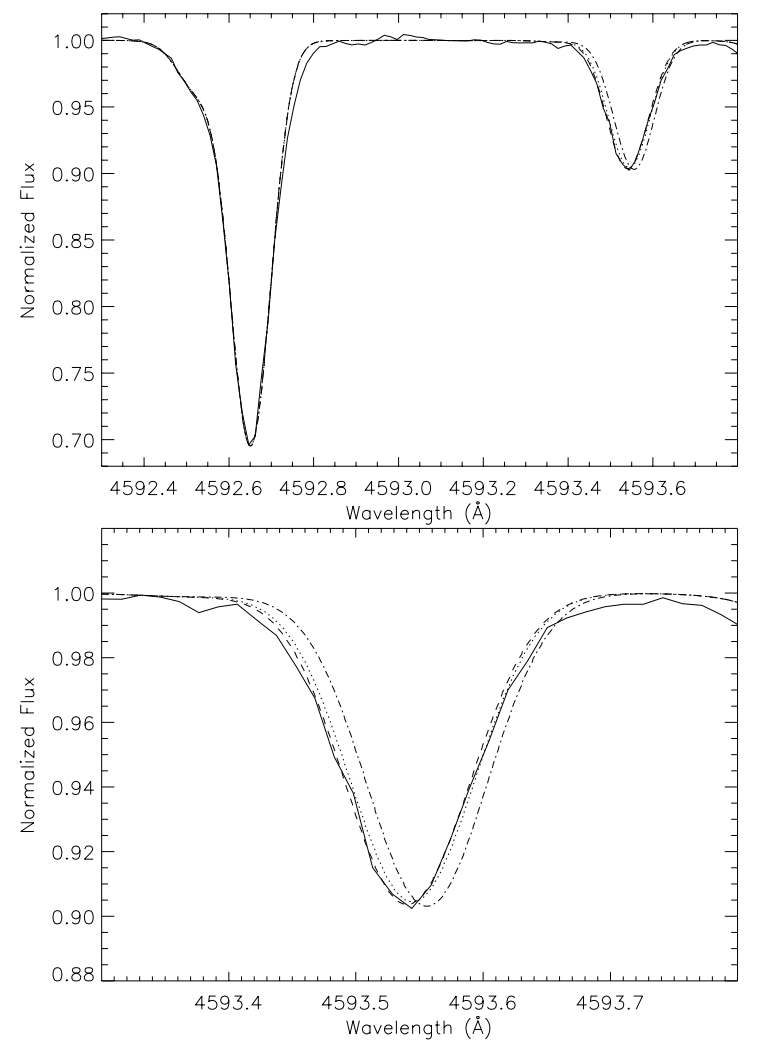

Fig. 5. Spectrum of a SmII line in the high $\mathrm{S} / \mathrm{N}$ data set of CS 31082-001. Upper panel: Fe I line at $4592.651 \AA$ together with the Sm II line at $4593.55 \AA$ A. Solid line is the observed spectrum, dotted line is a synthetic spectrum with solar system composition, dashed line is a synthetic spectrum with pure $r$-process composition and dash-dotted line is a synthetic spectrum with pure $s$-process composition. Lower panel: close up of the samarium line.

To create synthetic spectral lines for the case of the solar system isotope mixture the original single line in the line list was replaced by lines from each of the seven isotopes. The $g f$ value for each isotope was determined by scaling the transition $g f$ value by the percentage of the isotope in the solar system abundance. For the two odd isotopes the hfs was calculated and incorporated into the line list. The abundance we derive using the six lines shortward of $\lambda 4716$ is $\log \epsilon=-0.33 \pm 0.07$. The result of Hill et al. (2002) was $\log \epsilon=-0.51 \pm 0.16$. The difference is reasonable in light of our using updated $g f$ values (Lawler et al. 2006), having included IS and hfs, and using data of a higher $\mathrm{S} / \mathrm{N}$. To remove the $s$-process contribution of each isotope the isotope $g f$-value was scaled by the amount of its corresponding $s$-process fraction. A similar procedure was done for determining the $g f$-values for the $r$-process composition. Increasing the samarium abundance in the model then accounted for the differences in line depth with isotope mixture.

Examples of the line fitting are presented in Figs. 5 and 6. The strong line to the left in both figures is the Fe I line used for wavelength calibration and the line to the right is the Sm II line, which is enlarged in the lower panel. A close examination of the observed and the synthetic spectra gives an indication of a shift in the observed line similar to the shift of the profile indicative of a pure $r$ composition. The typical abundance difference, obtained from matching the depth of the lines, between the pure $r$ case and the solar system composition of the isotopes for any of our lines, is 0.15 dex. Between the solar system composition and the pure $s$ composition the difference is $0.47 \mathrm{dex}$. This difference can
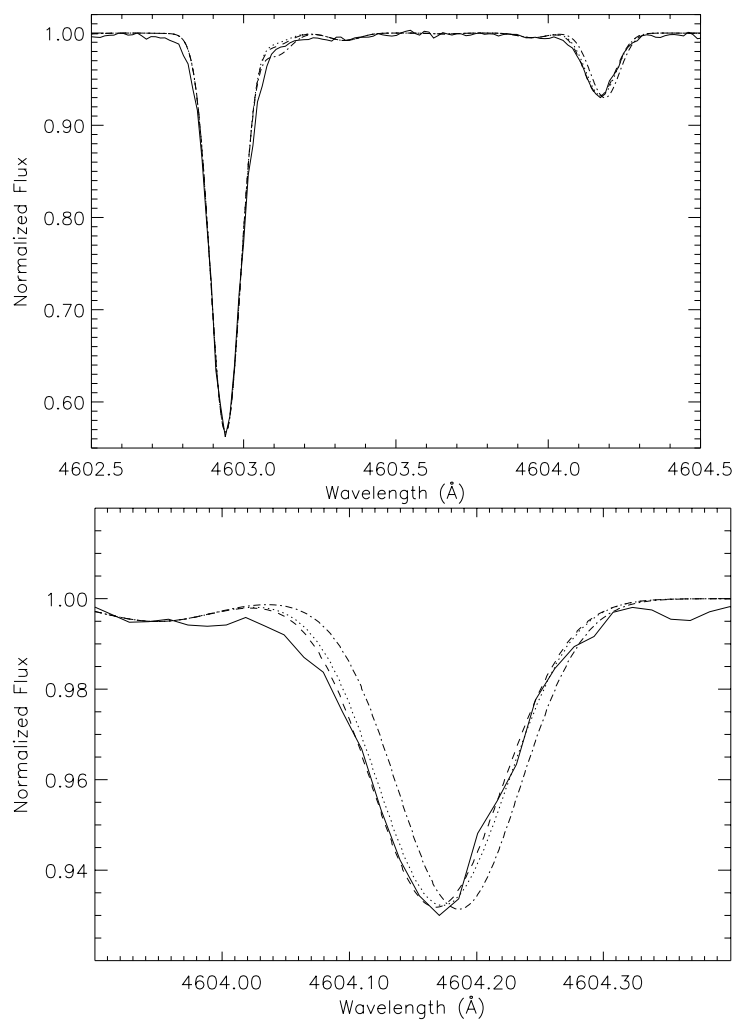

Fig. 6. Spectrum of an Sm II line in the high $\mathrm{S} / \mathrm{N}$ data set of CS 31082-001. Upper panel: Fe I line at $4602.941 \AA$ together with the Sm II line at $4604.17 \AA$ A. Lines are as in Fig. 5. Lower panel: close up of the samarium line.

also be seen in the wing of the synthesized $\lambda 4602.941 \mathrm{Fe}$ I line as there is a very weak Sm II line present. The measured wavelength of the Fe I line is unaffected by the blend. In Fig. 7 are presented the two Sm II lines at $\lambda \lambda 4591.82$ and 4595.29 , for which high $\mathrm{S} / \mathrm{N}$ spectra, hfs and IS data are available. The Sm II $\lambda \lambda 4715.27$ and 4693.63 lines are both blended with very weak Ti I lines, but are otherwise similar to $\lambda \lambda 4591.82$ and 4595.29 . By studying all six Sm II lines it can be noted that the wavelength shift between the pure $r$-process composition and the solar system composition is small and in most cases not even our high $\mathrm{S} / \mathrm{N}$ observed spectrum is sufficient to distinguish between the two profiles; however, for the lines with the largest IS the comparison between the observed and the synthetic spectra suggests an $r$-process dominated isotope mixture. It should also be noted that in the event of an $s$-process dominant isotope mixture the line shift is more easily distinguished and cannot fit the Sm II lines for CS31082-001.

In Figs. 8-10 three Sm II lines with TIS $_{148-154}$ as large as $0.3 \mathrm{~cm}^{-1}$ are shown. The $\lambda 5069.47$ line has known hfs constants, which the other two have not. As can be seen, even at this lower $\mathrm{S} / \mathrm{N}$ it is easy to rule out the case of a relative $s$-process enhancement. For the lines $\lambda \lambda 5052.75$ and 5103.09 the positions of the center-of-gravity for solar system composition (sun) of the samarium isotopes and a pure $r$-process composition (r) are labeled. The solar system isotope mixture center-of-gravity wavelength is measured in the laboratory spectrum while the pure $r$-process center of gravity position is estimated from comparisons with lines having known hfs constants as the simulation shown in Fig. 4. The Sm II $\lambda 5103.09$ line has a Ni I blend in its blue wing but it is possible to account for it in the synthetic spectrum. The line at $\lambda 5104.47$ listed in Table 4 is similarly weak as that at $\lambda 5103.09$ with a suspected weak blend in the red wing. 

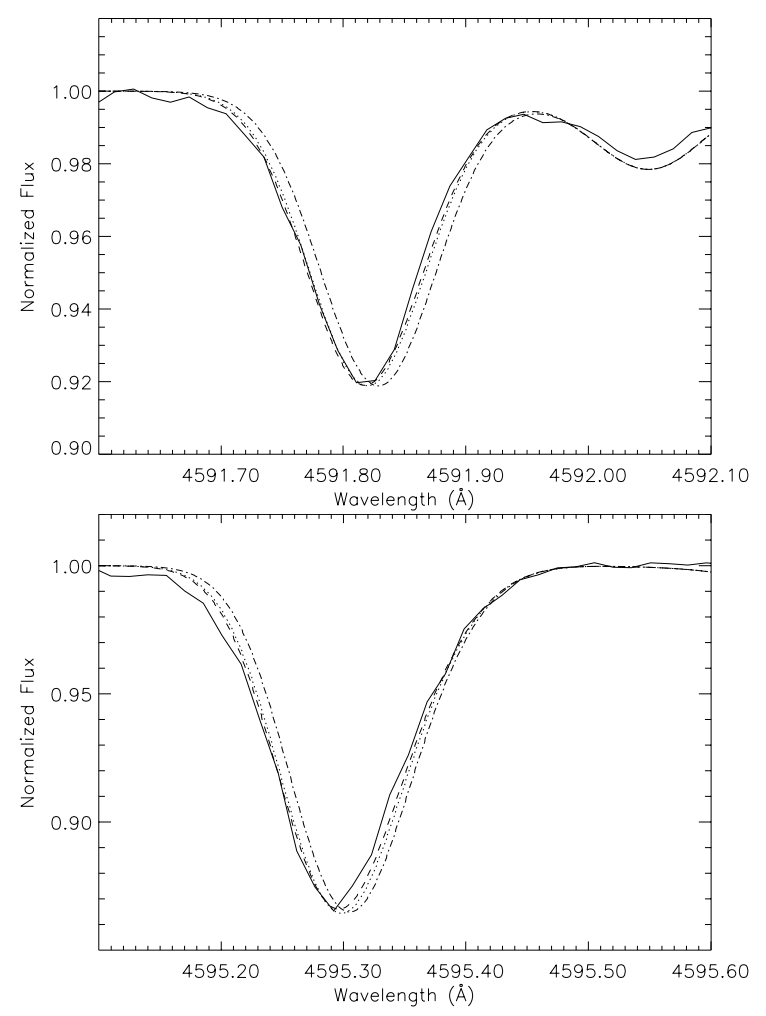

Fig. 7. Spectra of Sm II lines ( $\lambda \lambda 4591.82$ and 4595.29) in the high S/N data set of CS 31082-001. Lines are as in Fig. 5.

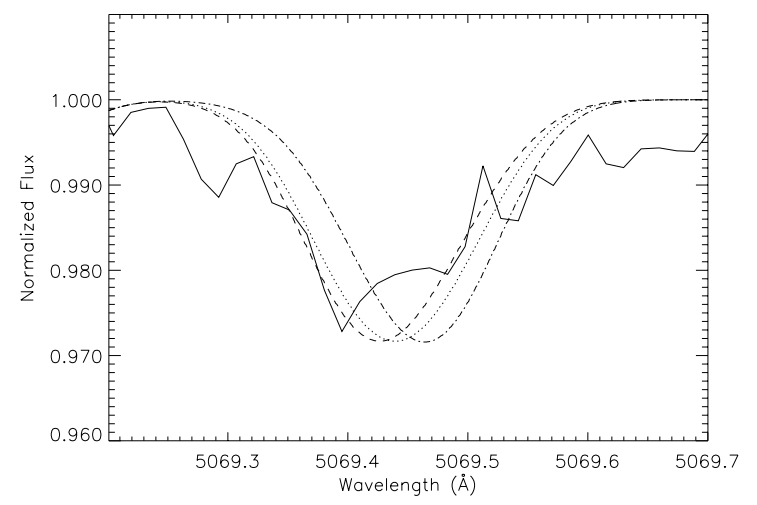

Fig. 8. The Sm II $\lambda 5069.47$ line having a very broad IS of $\mathrm{IS}_{148-154}=$ $0.303 \mathrm{~cm}^{-1}$. Lines are as in Fig. 5.

As can be seen for these lines the $\mathrm{S} / \mathrm{N}$ is not good enough for any detailed analysis at this point and there is a lack of hfs data, but with better data these lines have a great potential to be used for analysis of the isotope composition of samarium.

\section{Discussion}

The spectroscopic analysis undertaken in this work requires a high level of precision at all steps and places a higher demand upon precision than is typical in an abundance analysis. The level of precision needed for the measurement of line shifts between cases of a solar system isotope mixture and a pure $r$-process mixture is about $10 \mathrm{~m} \AA$ for lines with a $\mathrm{TIS}_{\mathrm{A}=154-148}$ of approximately $0.2 \mathrm{~cm}^{-1}$. For a shift of $10 \mathrm{~m} \AA$ it is only possible to determine whether the isotope mixture is either of these two cases, as it is currently not possible to within any credible accuracy deduce a specific isotope mixture. To do so would

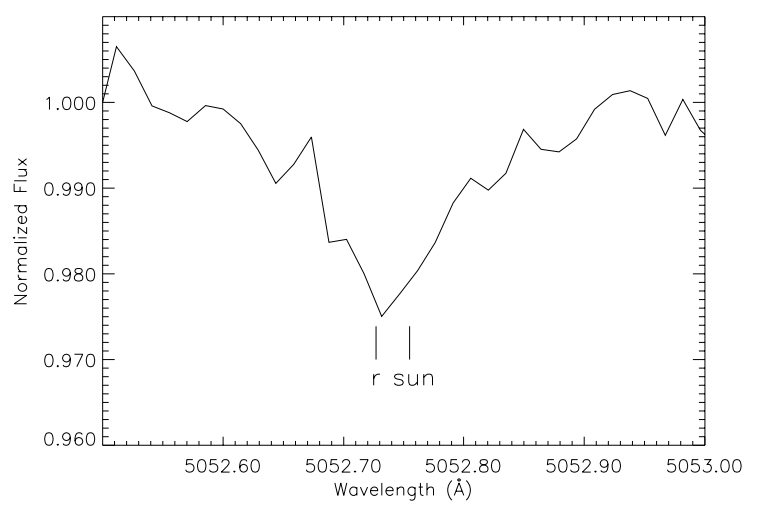

Fig. 9. The Sm II $\lambda 5052.75$ line with approximate center-of-gravity wavelengths marked for the solar system composition (sun) and the pure $r$-process (r).

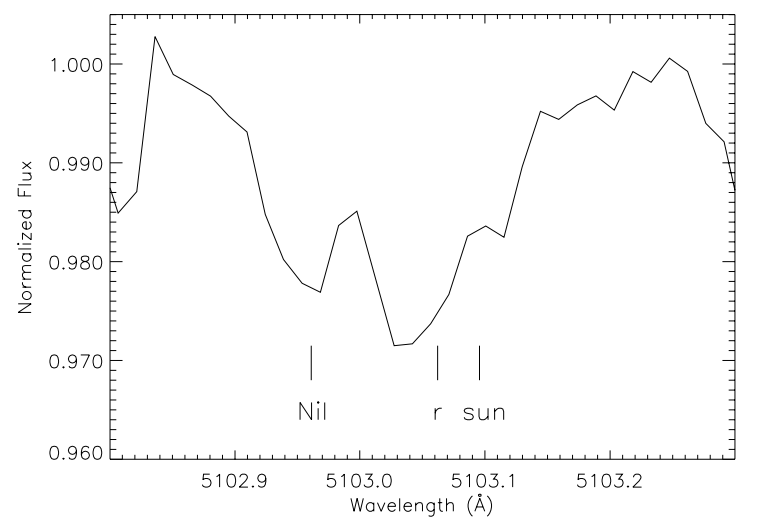

Fig. 10. The Sm II $\lambda 5103.09$ line with approximate center-of-gravity wavelengths marked for the solar system composition (sun) and the pure $r$-process (r).

require that the precision be increased by an order of magnitude. To apply the variation in line width to identifying isotope mixture requires a similar precision. At essentially every step of our investigation we have encountered limitations and we discuss here our attempts to deal with them and to outline how they can be overcome in the future.

The two sets of observations we have had at our disposal were both obtained primarily for abundance analyses. The lower $\mathrm{S} / \mathrm{N}$ dataset has been used for the extensive analysis of Hill et al. (2002). The red spectrum utilized here was used only for very few lines in that paper, which relied mainly on the bluest spectrum available at that time (3000-3800 ̊) and had a higher S/N. The high-S/N dataset has previously been used by Plez et al. (2004) to extract the abundance of lead. The lower S/N set is of insufficient quality to be useful in our current investigation, but it is the only available data for lines longward of $5000 \AA$. The high- $\mathrm{S} / \mathrm{N}$ dataset is sufficient for our preliminary investigation but is on the limit of what is useful for the samarium lines with $\mathrm{IS}_{\mathrm{A}=154-148}$ of about $0.2 \mathrm{~cm}^{-1}$. In Fig. 11 we present two examples of the difference between these two observed spectra to illustrate that improvements in $\mathrm{S} / \mathrm{N}$ for the lines longward of $5000 \AA$ would be likely to produce a useful constraint for determining the relative $r$ - and $s$-process contributions from the line profiles.

Spectral resolution is important for precision spectroscopy, but may be of secondary importance to the S/N since turbulent motions in the stellar atmosphere along with rotation velocity place limitations on spectral resolution. One potential 


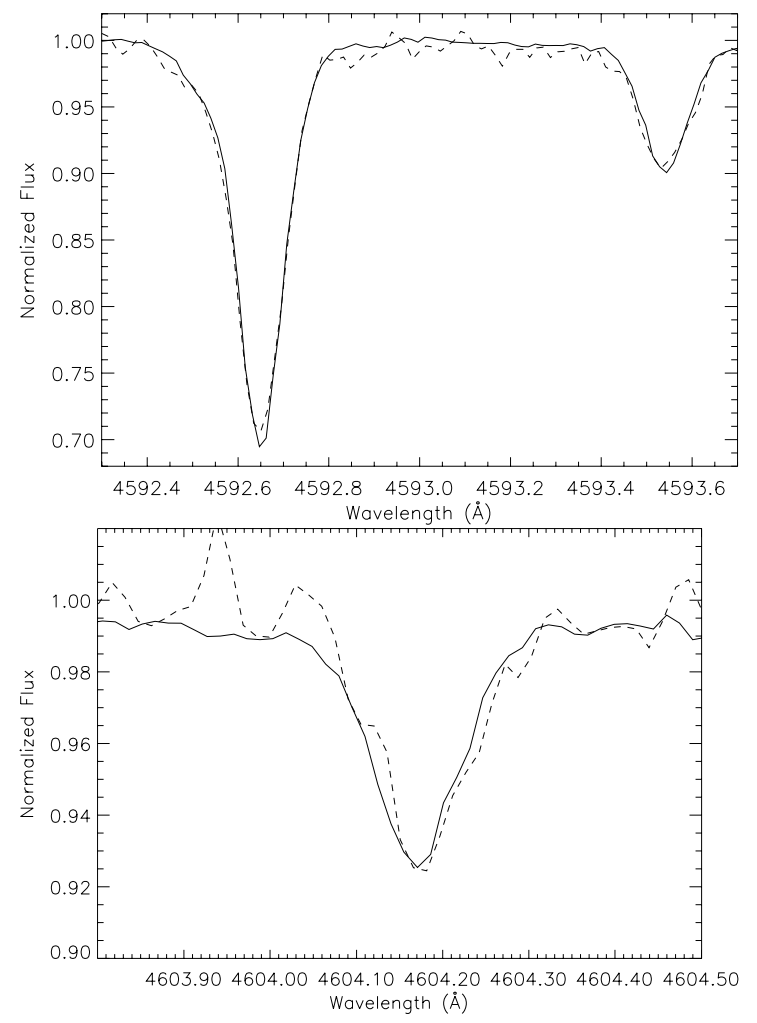

Fig. 11. Comparison of the influence of $S / N$ upon the two observed spectra. The solid line is the data set with $\mathrm{S} / \mathrm{N}$ of up to 600 and the dashed line is the data set with $\mathrm{S} / \mathrm{N}$ of up to 250 .

approach to increasing the accuracy of line profile analysis, without increasing the resolution of the instrument, would be to oversample the line profile. Such an approach was available on the Hubble Space Telescope's GHRS instrument, which shifting the spectrum by approximately one-quarter pixel for up to four subexposures. Currently, the VLT/UVES instrument also has the capability to shift the spectrum along the dispersion direction, but this feature was not used in acquiring the data used in this study.

The wavelength calibration of the stellar spectrum also plays a role in producing high quality spectra. Both datasets used here were reduced using standard pipeline processing (Ballester et al. 2000), which utilizes a Th-Ar lamp for wavelength calibration. This lamp has been compared with the High Accuracy Radial velocity Planet Searcher (HARPS), mounted on the ESO $3.6 \mathrm{~m}$ telescope at the La Silla, by Chand et al. (2006), who report a dispersion of $\sim 1 \mathrm{~m} \AA$ in the comparison between the two, which is within the stated accuracy of $\sim 4 \mathrm{~m} \AA$ for UVES. The stated accuracy of HARPS is less than $1 \mathrm{~m} \AA$ and therefore the wavelength accuracy of UVES might be even better than $4 \mathrm{~mA}$. This low an uncertainty for UVES is supported by our comparison with the Fe I lines having high accuracy laboratory wavelengths, which all were found to lie within $1 \mathrm{~m} \AA$ of the lines in the UVES spectra. There is though a subtle point that is not considered by Chand et al. (2006). The argon line list used for both the UVES and the HARPS wavelength calibration is that of Norlén (1973). This line list is suspected to have a small systematic error $\left(\sim 7\right.$ parts in $\left.10^{8}\right)$ because of the use of an external calibration source together with the hollow cathode for the calibration of the FTS (Whaling et al. 1995). This systematic error translates to $0.35 \mathrm{~m} \AA$ at $5000 \AA$ which should be considered when accuracies below $1 \mathrm{~m} \AA$ are desired. This is mostly of concern in the reddest part of the spectra where the Th spectrum is poor. In the region where our lines reside (4600-4700 ^) this should not be of any greater concern and we are also fortunate to have been able to find Fe I lines with high wavelength accuracy in close proximity to the Sm II lines.

Another concern of the reduction process is the normalization and splicing of the echelle orders. The algorithms used for these tasks can lead to breaks between the orders, which can make the wavelength scale of the concatenated spectrum less accurate over wavelength intervals that include more than one order. A skew to the line profile can also result from an improper normalization. This problem can be lessened by working in the order space and not merge the orders.

Likewise, high precision atomic data are needed for this investigation, i.e. wavelengths, IS, hfs constants, energy levels and $g f$ values. These parameters are not only needed for Fe I and Sm II but also for possible blends, both atomic and molecular, although the demands on precision are not as great on the blends as long as they are much weaker than the feature under study. Absolute wavelengths of spectral lines can be determined to sub$\mathrm{m} \AA$ precision through laboratory analysis using i.e. a FTS, especially if the lines have little or no structure. If necessary, relative wavelengths for components of isotope and hyperfine structure may be measured in some cases using laser techniques with uncertainties less than $0.1 \mathrm{~m} \AA$. The absolute wavelength can then be measured, as we have done, using a FTS. We have measured the position of the heaviest isotope of Sm II with an accuracy of $\sim 0.7 \mathrm{~m} \AA$ at $5000 \AA$, and higher accuracy FTS data may be possible. The current accuracy is significantly higher than that of the stellar spectra, as is desired.

The accuracy of the energy levels and the $g f$ values are not critical to our analysis, as long as the former are not used to determine wavelengths. However, if there are blending lines these need to have $g f$ values of a relatively high accuracy along with a well determined elemental abundance to make the samarium line useful. Since the blending line in most cases is likely to be narrower than the samarium line structure the blending may influence the result of the samarium isotope composition. Unknown blends will always be of great concern but as long as one is using several samarium lines possible blends might be detected as deviations in the results.

The model atmosphere might be of some concern when dealing with this kind of high precision measurements. ATLAS12 models are plane-parallel 1D models which do not account for i.e. NLTE, sphericity and turbulence effects addressed by multidimensional modeling. The most important of these effects for this study is likely that of turbulent motions. High resolution spectra of the sun clearly show asymmetric features and line shifts attributed to convective motions. These effects have not been detected in our data, although some iron lines appear slightly skewed, the troughs being slightly redshifted relative to the line-wing symmetry. The other two effects, NLTE and sphericity, should have a negligible effect on the wavelength of the lines, but might affect the strengths. As all isotope components of the Sm II lines under study are very weak, being within a few percent of the continuum, we would not expect their relative line strengths to be altered by the modeling.

Two factors affect the precision of the synthetic spectrum provided by the SYNTHE code: the input precision of the wavelengths (limited to $1 \mathrm{~m} \AA$ ) and the computational resolution of the synthetic spectrum (computation point spacing). The typical default computational resolution used for abundance analyses is 500000 , which translates to a precision of $10 \mathrm{~m} \AA$ at $5000 \AA$. This was deemed to be insufficient for our study and through a 
minor change to the code we increased it to allow one additional digit. We employed a computational resolution of $9 \times 10^{6}$, which translates to a precision of $0.55 \mathrm{~m} \AA$ at $5000 \AA$.

\section{Conclusion}

An investigation into the isotope structure of samarium and its usefulness in quantifying the isotope composition observed in stellar photospheres, as produced by the $r$ - and $s$-processes, has been conducted. We have measured the TIS of 11 lines of Sm II from laboratory FTS spectra. The TIS of the seven samarium isotopes is large for these lines, varying from TIS $_{154-148}=0.118$ to $0.318 \mathrm{~cm}^{-1}$. For lines with such large TIS our FTS data are sufficient for measuring the TIS of the even isotopes, giving results applicable to stellar spectrum analysis. Earlier measurements of IS done with laser methods are of higher accuracy and were incorporated into the analysis.

From spectral line analysis, the isotope mixture provides indicators of the $r$ - versus $s$-process contribution to the samarium content via line shifts and variations in line width and profile. The strongest indicator is the shift of the center of gravity of the line towards line components of the heavier isotopes when the isotope composition is changed from a solar system mixture to one more dominated by the $r$-process. In the case of a pure $r$-process mixture, this shift is on the order of $10 \mathrm{~m} \AA$ or larger for the lines investigated in this work and demands high wavelength accuracy in the observed spectrum. We used Fe I lines from our FTS spectrum as wavelength calibration when possible.

Using the high resolution, high $\mathrm{S} / \mathrm{N}$ spectrum of the metalpoor halo star CS 31082-001 we fitted synthetic spectra to six Sm II lines and can conclude that the lines with the largest IS $\left(\sim 0.2 \mathrm{~cm}^{-1}\right)$ show a slight shift in the same direction and amount as expected if the stellar samarium abundance is dominated by $r$-process produced isotopes. Even though these Sm II lines do not give a definitive quantitative result, indications for a line shift are present. Unfortunately, the available UVES spectrum above $5000 \AA$, where the lines with the largest IS are found, is not of sufficient $\mathrm{S} / \mathrm{N}$ for our purposes. We estimate that a $\mathrm{S} / \mathrm{N}$ ratio of more than 500-600 is needed to analyze the Sm II line profiles for isotope mixture, a $\mathrm{S} / \mathrm{N}$ that is well within reach of modern day instrumentation.

The large IS of Sm II provides for a method of determining the $r$ - versus $s$-process composition of a stellar photosphere which is independent of a many-element abundance analysis. The method can be used for stars in which the Sm II lines having large IS are unblended and the observed spectrum is of sufficient quality. The Sm II lines analyzed in this study would not be useful in isotope analyses of stars having solar abundances for irongroup elements, since increased line blending would prohibit finding weak, isolated Sm II lines. The center-of-gravity line shift is larger when changing from a solar system isotope mixture composition to a pure $s$-process mixture than when changing to a prominent $r$-process mixture. An interesting application of the current study would be to search for Sm II line shifts in the spectrum of the metal-deficient CH star CS 31062-050 Johnson $\&$ Bolte (2004), to confirm whether the observed abundance pattern is the result of the $s$-process.

\section{References}

Anders, E., \& Grevesse, N. 1989, Geochim. Cosmochim. Acta, 53, 197 Arlandini, C., Käppeler, F., Wisshak, K., et al. 1999, ApJ, 525, 886

Ballester, P., Modigliani, A., Boitquin, O., et al. 2000, The Messenger, 101, 31 Beiersdorf, S., Heddrich, W., Kesper, K., et al. 1995, J. Phys. G, 21, 215

Biémont, E., Garnir, H. P., Litzén, U., et al. 2003, A\&A, 399, 343

Chand, H., Srianand, R., Petitjean, P., et al. 2006 [arXiv: astro-ph/0601194] Edlén, B. 1966, Metrologia, 2, 71

Ekeland, A., \& Hauge, O. 1975, Sol. Phys., 42, 17

Hill, V., Plez, B., Cayrel, R., et al. 2002, A\&A, 387, 560

Ivarsson, S., Andersen, J., Nordström, B., et al. 2003, A\&A, 409, 1141

Johnson, J. A., \& Bolte, M. 2004, ApJ, 605, 462

King, A. S. 1935, ApJ, 82, 140

Kurucz, R. 1993, SYNTHE Spectrum Synthesis Programs and Line Data. Kurucz CD-ROM No. 18 (Cambridge, Mass.: Smithsonian Astrophysical Observatory)

Kurucz, R., \& Bell, B. 1995, Atomic Line Data (R.L. Kurucz and B. Bell) Kurucz CD-ROM No. 23 (Cambridge, Mass.: Smithsonian Astrophysical Observatory)

Lambert, D. L., \& Allende Prieto, C. 2002, MNRAS, 335, 325

Lawler, J. E., Den Hartog, E. A., Sneden, C., \& Cowan, J. J. 2006, ApJS, 162, 227

Learner, R. C. M., \& Thorne, A. P. 1988, JOSA, B5, 2045

Lundqvist, M., Nilsson, H., Wahlgren, G. M., et al. 2005, A\&A, 450, 407

Magain, P. 1995, A\&A, 297, 686

Magain, P., \& Zhao, G. 1993, A\&A, 268, L27

Mashonkina, L., \& Zhao, G. 2005, in From Lithium to Uranium: Elemental Traces of Early Cosmic Evolution, ed. V. Hill, P. Francois, \& F. Primas (Cambridge: Cambridge Univ. Press), Proc. IAU Symp., 228, 491

Masterman, D., Rosner, S. D., Scholl, T. J., Sharikova, A., \& Holt, R. A. 2003, Can. J. Phys., 81, 1389

Norlén, G. 1973, Phys. scr., 8, 249

Plez, B., Hill, V., Cayrel, R., et al. 2004, A\&A, 428, L9

Rao, P. M., Ahmad, S. A., Venugopalan, A., \& Saksena, G. D. 1990, Z. Phys. D, 15,211

Rosman, K. J. R., \& Taylor, P. D. P. 2004, J. Phys. Ref. Data, 27, 1275

Sneden, C., Cowan, J. J., Lawler, J. E., et al. 2002, ApJ, 566, L25

Sneden, C., Cowan, J. J., Lawler, J. E., et al. 2003, ApJ, 591, 936

Villemoes, P., Wang, M., Arnesen, A., Weiler, C., \& Wännström, A. 1995, Phys. Rev. A, 51, 2838

Wahlgren, G. M. 2002, Phys. scr., T100, 22

Wahlgren, G. M. 2005, Mem. S. A. It. Suppl., 8, 108

Westin, J., Sneden, C., Gustafsson, B., \& Cowan, J. J. 2000, ApJ, 530, 783

Whaling, W., Anderson, W. H. C., Carle, M. T., Brault, J. W., \& Zarem, H. A. 1995, J. Quant. Spec. Radiat. Transf., 53, 1

Young, L., Childs, W. J., Berry, H. G., Kurtz, C., \& Dinneen, T. 1987, Phys. Rev. A, 36, 2148 